\title{
Overview Of Application Of Traffic Simulation Model
}

\author{
Nurul Nasuha Nor Azlan", Munzilah Md Rohani',** \\ ${ }^{1}$ Faculty of Civil and Environmental Engineering, Universiti Tun Hussein Onn Malaysia, Parit Raja, 86400, Johor, Malaysia \\ ${ }^{2}$ Faculty of Civil and Environmental Engineering, Universiti Tun Hussein Onn Malaysia, Parit Raja, 86400, Johor, Malaysia
}

\begin{abstract}
Traffic simulation is a widely used method applied in the research on traffic modelling, planning and development of traffic networks and systems. From the literature study, a variety traffic simulation models were found in experiments and applications with aims to imaginary real traffic operations. The traffic simulation models can be categorised into three namely, microscopic modelling, macroscopic modelling and mesoscopic modelling. This report is aimed to overview these traffic simulation models, in term of its function, limitation and application.
\end{abstract}

\section{Introduction}

Traffic modelling is aimed to accurately recreate traffic as observed and measured on street. Traffic modelling assumed the appearance of a traffic system without replicating. It was developed based on modeller's experience that integrate the mathematical models into traffic system [1]. Traffic modelling plays an important part in traffic engineering. It can be applied to plan and manage the traffic within certain road network [2]. For example, making a smooth traffic at an intersection and etc [3].

Principally, simulation models focus on three output values to solve traffic problems [4]. First is the traffic flows. In traffic flows, alternative routes can be identified based on the number of vehicles. By using the simulation model, modeller can devise on how to reduce the levels of congestion of certain roads. Second output is the network element. Network element in traffic simulation consists of link, merge, link cross and other elements of the road [5]. This is related to the geometric layout of the road. Using appropriate simulation software, the road geometric design can be changed to see how it can influence the current traffic situation. Third output is the skim category. Simulations model can help to estimate the time and cost of travel. This is especially used when the assessment of traffic improvement is needed to be measured. The transport planner can easily make a performance comparison without any extra cost of money and time.

\section{Classification of Traffic Simulation Models}

The models were grouped according to their areas of application. The three classification of traffic simulation models, are microscopic modelling, macroscopic modelling and mesoscopic modelling.

\subsection{Microscopic Modelling}

Microscopic modelling based on the characteristics of various vehicle movements such as cars, buses, motorcycles and so on in the traffic flow. Microscopic modelling aimed to collect data parameters, such as, flow, density, speed, travel and delay time, long queues, stops, pollution, fuel consumption and shock waves. The characteristics of microscopic modelling methods were based on car-following model, lane-changing models and gaps of the individual drivers.

\subsubsection{Car Following Models}

These algorithms introduced a concept that a driver recognize and follow a lead vehicle in a lower speed [6]. This can be described as a situation such as in a car platoon without being able to change the lane. Generally, the following car algorithm determined the relationship with the lead vehicle at times. It was the function of spacing, speed and acceleration at times. The closer the following vehicle to the lead vehicle the more sensitive the reaction of the following vehicle to the lead vehicle. This sensitivity also increases with speed. This is because if the main vehicle was driven in a lower speed, the following vehicles will then reduce the speed. This resulted the occurrence of car platoon and traffic congestion.

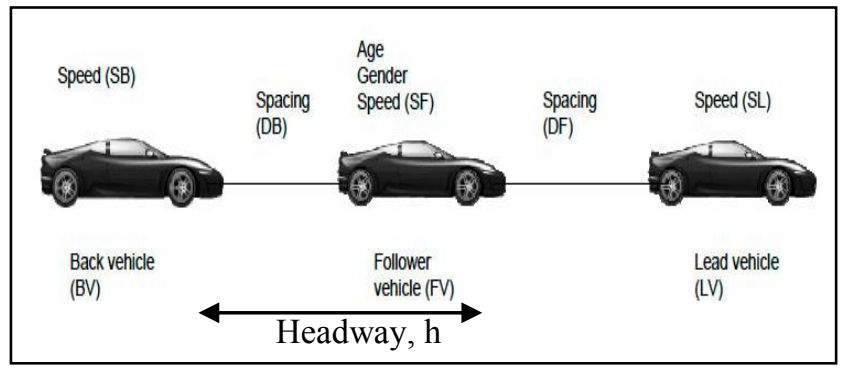

Fig. 1. Car Following Model

\footnotetext{
* Corresponding author: munzilah@uthm.edu.my
} 
In the figure above, headway (h) is the horizontal distance between two vehicles. Spacing (s) is the distance measured from the rear-bumper of the following vehicle to rear-bumper of the lead vehicles pass at a certain point within time interval. The time headway is defined as the time difference of successive vehicles crossings at the point. Microscopic modelling is based on the movement of individual vehicles and their relative time and space.

\subsubsection{Lane Changing Models}

Gipps has proposed a development of the lane-changing model [7]. The lane-changing model is a decision process to estimate the driver's behaviour in making a lane change within the time given. Lane changing can be categorised into two types. Mandatory lane change, which occurs when a driver change lane to a specified lane. For instance, a driver changed to the right lane when he wanted to make a right turn at the next intersection and discretionary lane change, which the driver made to the next lane to avoid following trucks and increase speed.

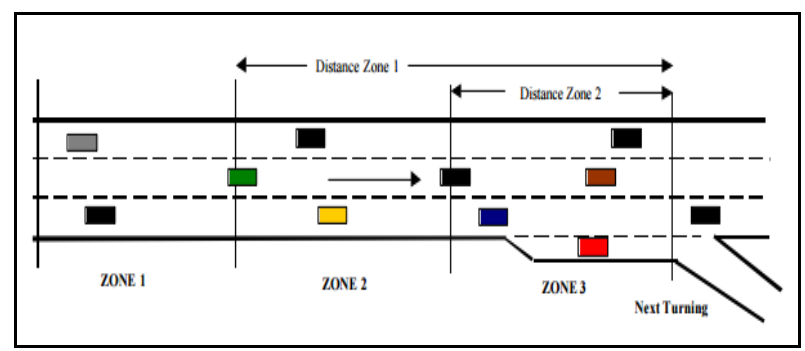

Fig. 2. Lane Changing Zones

- Zone 1 : The furthest distance from the next turning to measure how the driver:

i. Change vehicles lane and speed of driver

ii. Speed and distance of lead vehicles

iii. Speed and distance upcoming lead vehicles at the other lane

- Zone 2 : The intermediate zone. The vehicles is looking for a gap to turn into the turning lane which affects the lane-changing decision.

- Zone 3 : This is the shortest distance to the next turning point. The vehicle has started to change lane at the merging lane to the next turning. It is necessary to reduce the speed to provide a big gap for vehicle to change the lane.

\subsubsection{Gap Acceptance Models}

Gap acceptance are used to determine the number of vehicles of traffic that can pass through a conflict point. A gap is defined as the time headway in between the lead and lag vehicles in the target lane. The lead gap is the gap to the lead vehicle in the target lane. The lag gap is the gap to lag vehicle in the target.

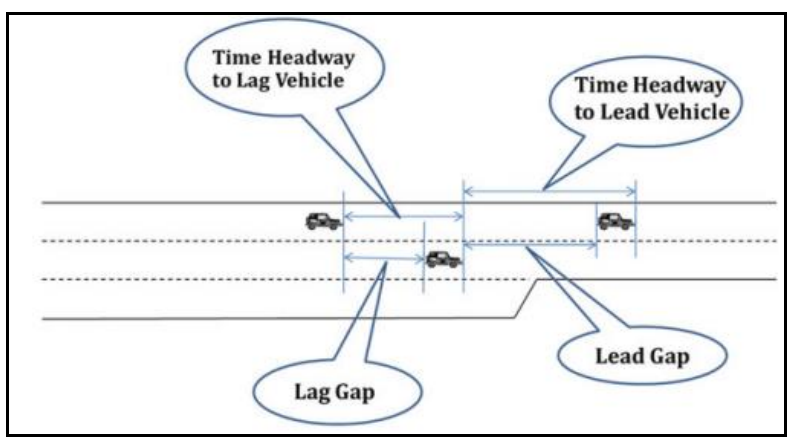

Fig. 3. Lane Changing with Consideration of Lead and Lag gaps

Gap acceptance models were used to determine the size of gap that will be accepted or rejected by a driver who aimed to merge or cross the intersection. Critical gap is defined as the number of accepted gap shorter than its equal to the number of rejected gaps. The driver needs time to clear the intersection and decide. The parameters of the gap acceptance model are the acceleration rate, desired speed and speed acceptance. Among these, the acceleration rate, the maximum give-way time and visibility distance at the intersection are the most important. The acceleration-rate is the ability of the vehicle to accelerate with required safety gap. The maximum give-way time is to determine when a driver starts to get intolerant if they cannot identify the gap.

\subsection{Macroscopic Modelling}

Macroscopic modelling describes the intersections at a low level of detail [8]. In macroscopic model, traffic stream is represented in an aggregate measured in terms of characteristics like speed, flow and density. Flow, speed and density are the three main characteristics of traffic. The researchers have studied speed-flow-density relationships and have attempted to develop mathematical descriptions for these curves, which is Greenshield's Model and Greenberg Model. Greenshield's Model is used to develop a model of uninterrupted traffic flow [9] It is a fairly accurate and simple modelling. There are three characteristics of the graph.

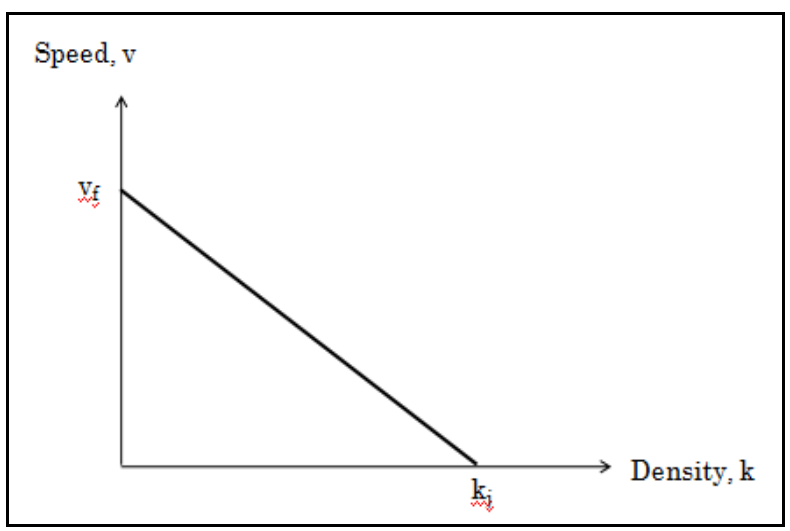

Fig. 4. Speed, v versus Density, k 
Greenshied's Model shows the relationship between speed and density is linear. When density increases, flow decreases to an optimum when more vehicles were on the road. The speed also decreases due to the interaction of vehicles.

$$
v=v_{f}-\frac{v_{f}}{k_{j}} k
$$

Where,

$\mathrm{v}=\operatorname{speed}(\mathrm{km} / \mathrm{h})$

$\mathrm{k}=$ density $(\mathrm{veh} / \mathrm{km})$

$\mathrm{v}_{\mathrm{f}}=$ free mean speed $(\mathrm{veh} / \mathrm{km})$

$\mathrm{k}_{\mathrm{j}}=\mathrm{jam}$ density $(\mathrm{veh} / \mathrm{km})$

Eq. 1. Speed, $v$

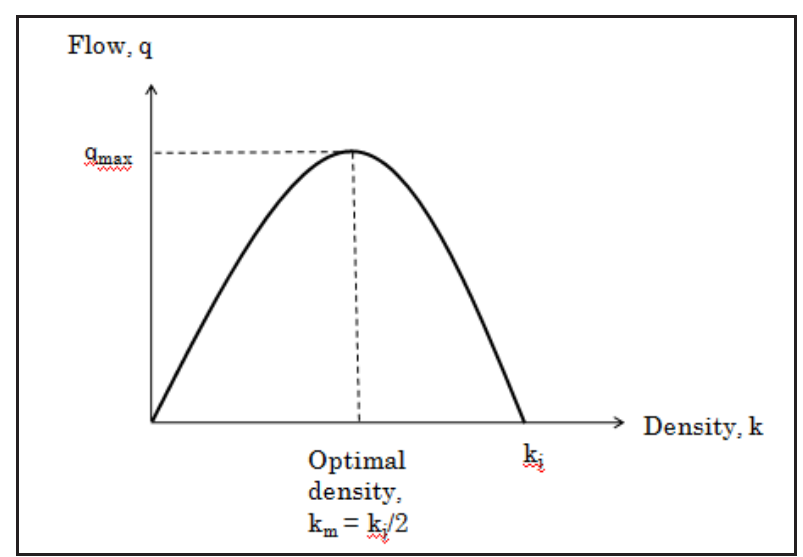

Fig. 5. Flow, $q$ versus Density, $k$

Greenshield's model shows that the relationship between flow and density is a parabolic curve.

$$
q=\left(v_{f}-\frac{v_{f}}{k_{j j}} k\right) k
$$

Where,

$q=$ flow

Eq. 2. Flow, $q$

When flow is very low, speed is higher. The drivers are able to travel at a desired speed. As the flow increases, speed gradually decreases. The highest flow shows the transition of non-congested to congested condition.

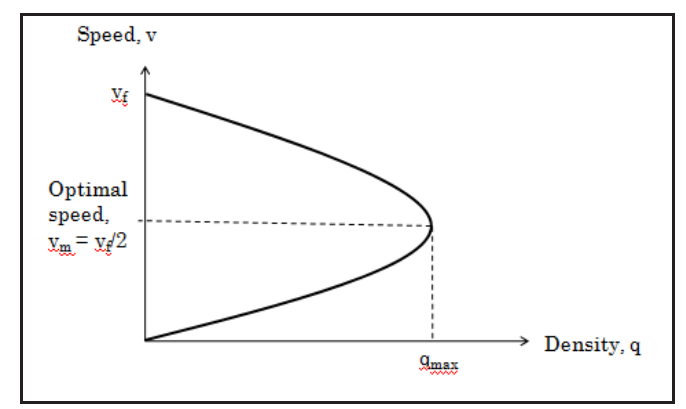

Fig. 6. Speed, $v$ versus Density, $k$
Greenshield's model shows the relationship between speed and density.

Where,

$$
q_{\mathrm{m}}=v_{\mathrm{m}} k_{\mathrm{m}} \text { or } q_{\mathrm{m}}=\frac{v_{f} k_{f}}{4}
$$

$\mathrm{q}_{\max }=$ maximum flow

$\mathrm{V}_{\max }=$ optimal speed

$\mathrm{k}_{\max }=$ optimal density

\section{Eq. 3. Maximum flow}

When the capacity $\left(\mathrm{q}_{\max }\right)$ is in the maximum flow, the density is increasing and the speed will decrease due to the maximum number of vehicles passing to a certain point. The characteristics of congested unstable flow is high density and low speed. There is no gap for vehicles to enter. The characteristics of uncongested stable flow is slow density and high speed, when there are gaps for merging lane.

\subsection{Mesoscopic Modelling}

Mesoscopic modelling describes the analysed transportation elements in small groups. Mesoscopic models are a combination of microscopic modelling and macroscopic modelling. In this model, platoon dispersion is stimulated. There are two methods of mesoscopic modelling which is platoon dispersion and vehicle platoon behaviour.

The first method is platoon dispersion. As a platoon moves downstream from an upstream intersection, the distance between the vehicle which may be due to the differences in the vehicle speeds, vehicle interactions (lane changing and other interferences), (parking, pedestrians and others). This phenomenon is called as platoon dispersion. The second method is vehicle platoon behaviour is a group of vehicles, travel at a short headway and moving at the same speed. Vehicle platoon behaviour is to predict the arrival of vehicle platoon with time, the total arrival time.

The important considerations in modelling of platoon behaviour are the number of vehicles in a platoon, speed of a platoon and the distribution of speed [10]. Platoon are formed when the traffic signal turns to red and the vehicles start to queue. For an isolated traffic signal, the lenght of queue is the key performance measures that is afftected by the vehicle arrival pattern [11]. The platoon will disperse when the traffic signal starts to change from yellow to green, so it starts with incerasing the speed, then the vehicles start to move. Once the vehicles are released from the stop line, they will start to move in a tight platoon position with short time headways and will start to disperse travel further downstream [12].

Table 1 presents the various software and modelling applied by previous research on traffic simulations. From the figure, it shows that the simulation modelling can solved a range of problems related to transportation particularly in public transport. 


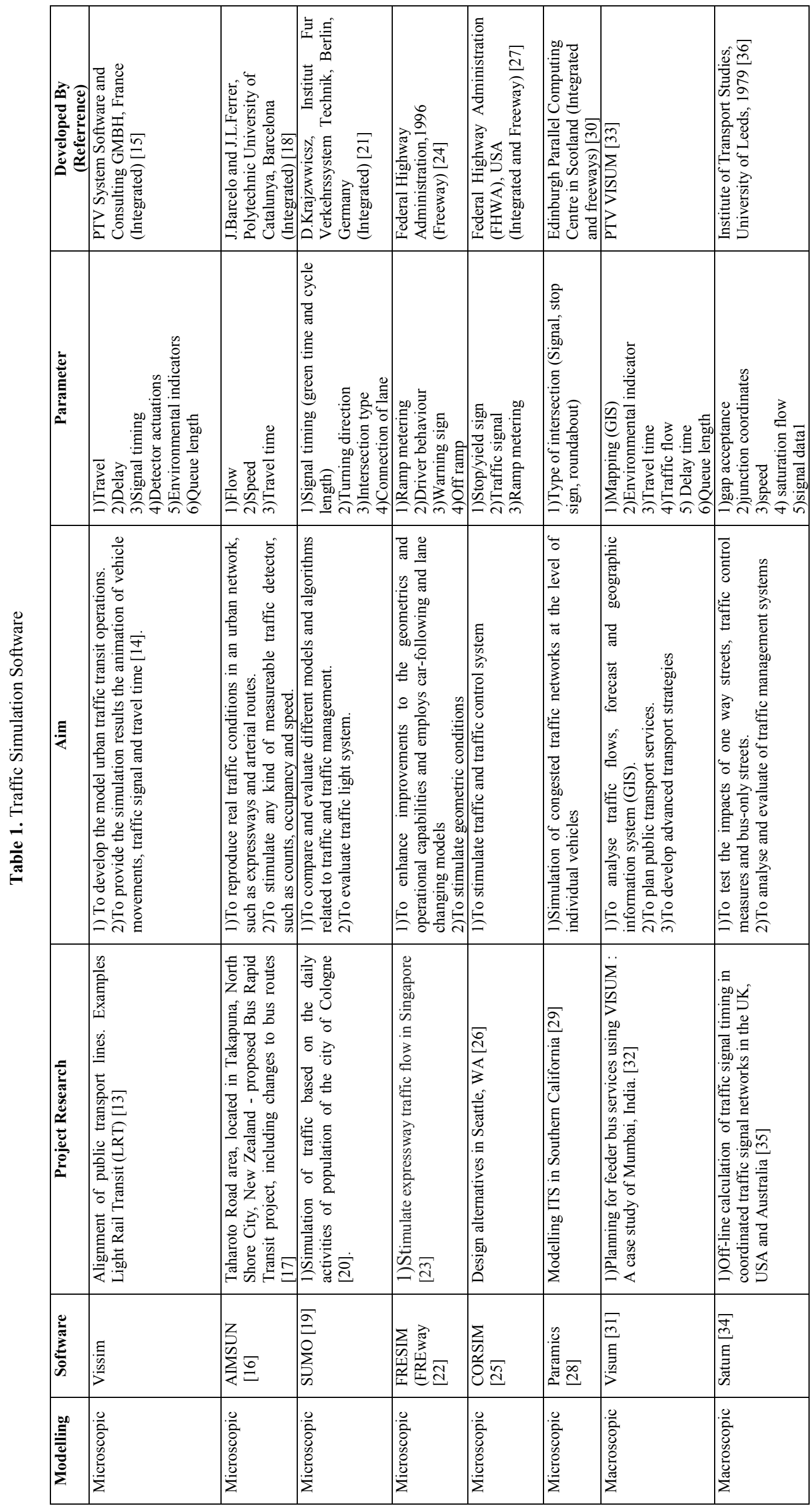




\section{Conclusion}

The objective of simulation model is to presents a real traffic situation in to dynamic model. There are three simulation model available to be used in traffic engineering namely macroscopic, microscopic and mesoscopic. The application on each model is depending on the aim of study to be conducted. Selecting the right model according to study aims is an important step towards traffic problem resolution.

The authors acknowledge the contribution of the Ministry of Malaysian of Higher Education (MOHE) and the University Tun Hussein Onn Malaysia (UTHM). This research is funded under FRGS Grant scheme (vot 1451) and GPPS Grant scheme (vot U778).

\section{References}

1. J.G. Shanthikumar, R.G. Sargent. A Unifying View Of Hybrid Simulation/Analytic Models And Modeling. Operations research,31(6), pp.10301052 (1983)

2. A. D. May, Traffic Flow Fundamentals, Prentice Hall Englewood Cliffs, New Jersey (1990).

3. S. Mahajan, A. Umadekar, K.Jethwa. New Concept Of Traffic Rotary Design At Road Intersections. Procedia-Social and Behavioral Sciences, 96, pp.2791-2799 (2013)

4. M. Friedrich. Multimodal Transport planning. Stuttgart University, Germany (2015). (Lecture Notes).

5. J. Barcello. Fundamentals of Traffic Simulation. International Series in Operations Research and Management Science. Barcelona, Spain, pp. 68-69 (2010)

6. R.M. Michaels. Perceptual Factors In Car Following. In Proceedings of The 2nd International Symposium on The Theory of Road Traffic Flow, London, England, OECD (1963)

7. P.G. Gipps. A Model For The Structure Of LaneChanging Decisions. Transportation Research Part B: Methodological, 20(5), pp. 403-414 (1986)

8. A.K. Rathi, Z.A. Nemeth. Freesim: A Microscopic Simulation Model Of Freeway Lane Closures (abridgment), 91, pp. 21-24 (1996)

9. B.D. Greenshields, W. Channing, H.A. Miller. A Study Of Traffic Capacity. In Highway research board proceedings. National Research Council (USA), Highway Research Board, 14, pp. 448-477 (1935)

10. H. Qing, L. Head, J. Ding. PAMSCOD: PlatoonBased Arterial Multi-Modal Signal Control With Online Data. Transportation Research Part C: Emerging Technologies, 20(1), pp. 164-184 (2012)

11. Highway capacity manual. Washington, DC (2000)

12. L. Yu. Calibration Of Platoon Dispersion Parameters On The Basis Of Link Travel Time Statistics. Transportation Research Record: Journal of the Transportation Research Board, 1727, pp. 8994 (2000).
13. R. Cervero. Journal Report: Light Rail Transit And Urban Development. Journal of the American Planning Association, 50(2), pp.133-147 (1984)

14. J. Barceló, E. Bernauer, L. Breheret, G. Canepari, C.D. Taranto, J. Ferrer, R. Liu. Simulation Modelling Applied to Road Transport European Scheme Tests (SMARTEST)-Review of MicroSimulation Models. In Institute for Transport Studies, University of Leeds (1998)

15. PTV VISSIM 5.30-05 User Manual. Karlsruhe: Planning Transport, Verkehr AG (2011)

16. J. Barceló, J. Casas. Dynamic Network Simulation With AIMSUN. Springer US. pp. 57-98 (2005)

17. C.P. Haas. Assessing Developments Using AIMSUN. In Institution of Professional Engineers New Zealand. Annual conference (2001)

18. J. Barcelo, L. Ferrer, R. Martin. Simulation Assisted Design and Assesment of Vehicle Guidance Systems. Department d'Estadistica I Investigacio Operativa, Polytechnic University of Catalunya, (1997)

19. M. Behrisch, L. Bieker, J. Erdmann, D. Krajzewicz. SUMO-Simulation of Urban Mobility: An Overview. In Proceedings of SIMUL 2011, The Third International Conference on Advances in System Simulation. ThinkMind (2011)

20. Hertkorn, G, Wagner, P. The Application Of Microscopic Activity Based Travel Demand Modelling In Large Scale Simulations (2004)

21. D. Krajzewicz, G. Hertkorn, C. Rössel, P. Wagner. SUMO (Simulation of Urban Mobility)-An OpenSource Traffic Simulation. In Proceedings of the 4th middle East Symposium on Simulation and Modelling, pp. 183-187 (2002)

22. H. Rakha, B. Crowther. Comparison And Calibration Of FRESIM And INTEGRATION Steady-Statecar-Following Behavior. Transportation Research Part A: Policy and Practice, 37(1), pp. 127 (2003)

23. R.L. Cheu, X. Jin, K.C. Ng, Y.L. Ng, D. Srinivasan. Calibration Of FRESIM For Singapore Expressway Using Genetic Algorithm. Journal Of Transportation Engineering, 124(6), pp. 526-535 (1998)

24. Federal Highway Administration. US Department of Transportation (1981)

25. A. Halati, H. Lieu, S. Walker. CORSIM-Corridor Traffic Simulation Model. In Traffic Congestion and Traffic Safety in the 21st Century: Challenges, Innovations And Opportunities (1997)

26. L. Bloomberg, J. Dale. Comparison of VISSIM and CORSIM traffic simulation models on a congested network. Transportation Research Record: Journal of the Transportation Research Board, 1727, pp. 5260 (2000)

27. Federal Highway Administration. US Department of Transportation (1981)

28. G.D.B. Cameron, G.I.D. Duncan. PARAMICS Parallel Microscopic Simulation Of Road Traffic. The Journal of Supercomputing, 10(1), pp. 25-53 (1996)

\footnotetext{
* Corresponding author: munzilah@uthm.edu.my
} 
29. B. Abdulhai, J.B. Sheu, W. Recker. Simulation Of ITS On The Irvine FOT Area Using" Paramics 1.5" Scalable Microscopic Traffic Simulator: Phase I: Model Calibration and Validation. California Partners for Advanced Transit and Highways (PATH) (1999)

30. M. Smith, G. Duncan, S. Druitt. PARAMICS: Microscopic Traffic Simulation For Congestion Management (1995)

31. C. Hildebrand, S.A. Hörtin. Comparative Study Between Emme And Visum With Respect To Public Transport Assignment. (2014)

32. B.R. Muley, U. Chande, C. S. R. K. Prasad. Planning For Feeder Bus Services Using Visum: A Case Study Of Mumbai, India (2015)

33. PTV VISUM User Manual (2014)

34. J. Aron. Using 'SATURN' To Determine The Effect Of Data On Model Accuracy And Operation Efficiency On A Network Of A Small CBD (Doctoral dissertation) (2014)

35. L. He, C. Fu, L. Yang, S. Tong, Q. Luo. Coordinated Real-Time Control Algorithm For Multi-Crossing Traffic Lights. In Natural Computation (ICNC), 2014 10th International Conference. Pp. 128-133. IEEE (2014)

36. T. Worsley, P. Mackie. Institute for Transport Studies, University of Leeds (2015) 